\title{
Feedback interference and dissociations of classification: Evidence against the multiple-learning-systems hypothesis
}

\author{
Roger D. Stanton And Robert M. Nosofsky \\ Indiana University, Bloomington, Indiana
}

\begin{abstract}
Researchers have argued that different categorization problems are learned by separate and distinct cognitive systems. They propose that an explicit system is responsible for learning rule-based categories and that a separate implicit system learns information-integration categories. One source of supporting evidence involves experiments in which observers perform a concurrent memory-scanning task that interferes with the processing of feedback. Researchers have reported a dissociation in which this manipulation impairs learning of a rule-based category but not an information-integration category. In the present research, we test the hypothesis that the dissociation was the result of lowered perceptual discriminability in the rule-based structure in comparison with the information-integration one. We demonstrate an example of an alternate rule-based category with easy-todiscriminate stimuli in which performance is unaffected by the interfering memory-scanning task. Furthermore, we demonstrate that learning of an information-integration category with low perceptual discriminability is impaired by the memory-scanning task. These demonstrations of the reverse dissociation challenge the interpretation that rule-based and information-integration category structures are learned by separate cognitive systems.
\end{abstract}

A fundamental question in the field of categorization is whether category learning is best described in terms of a single representational system or in terms of multiple systems (e.g., Ashby, Alfonso-Reese, Turken, \& Waldron, 1998; Erickson \& Kruschke, 1998; Nosofsky \& Johansen, 2000; Nosofsky \& Zaki, 1998). Within the class of singlesystem models, a variety of representational formats exist; however, most multiple-system models propose that category learning is mediated by an explicit system and at least one implicit system.

The COVIS (competition between a verbal and an implicit system) model (Ashby et al., 1998) is a prominent theory in category learning and is representative of the class of models that posit multiple systems. According to COVIS, there are two systems responsible for category learning: an explicit system that tests hypotheses on the basis of verbalizable rules, and an implicit system that is mediated by procedural learning. The explicit system relies on access to working memory and executive attention, using them to store and assess candidate rules. On each trial, a response is executed on the basis of the current rule. The observer continues to use the rule until corrective feedback suggests that the rule may be incorrect, and then the observer decides to either maintain the rule or search for a new rule. Given the latter option, a new rule must be selected and attention switched to this rule, and these processes require both time and effort. However, the implicit system is based on a procedural learning system, dependent on dopamine reward signals, that is updated automatically.

Recently, Maddox, Ashby, Ing, and Pickering (2004) sought to support the assumptions of the explicit and implicit learning components of COVIS. These researchers distinguish between two fundamental category types: rule-based categories and information-integration categories. Rule-based categorization problems are those in which it is easy for an observer to verbalize the optimal strategy. The observer selectively attends to each dimension, decides on the category regions along each of the component dimensions, and formulates a rule to determine category membership. An example is Maddox et al.'s unidimensional category structure, depicted in Figure $1 \mathrm{~A}$. The stimuli are sine wave gratings that vary in spatial frequency (perceived as bar width) and angle, and the vertical line represents the optimal decision bound for separating the categories. In this example, only spatial frequency determines category membership, and the angle of the gratings is irrelevant. Any percept left of the decision bound belongs to Category $\mathrm{A}$, and any percept right of the decision bound belongs to Category B. In general, for rule-based categories, decisions about the percept's value along each dimension are made first, and then these separate decisions are combined to generate a response. In the present case, only a single decision is necessary, and the simple verbal rule corresponds to "Respond A if the bars are wide and respond B if the bars are thin." 


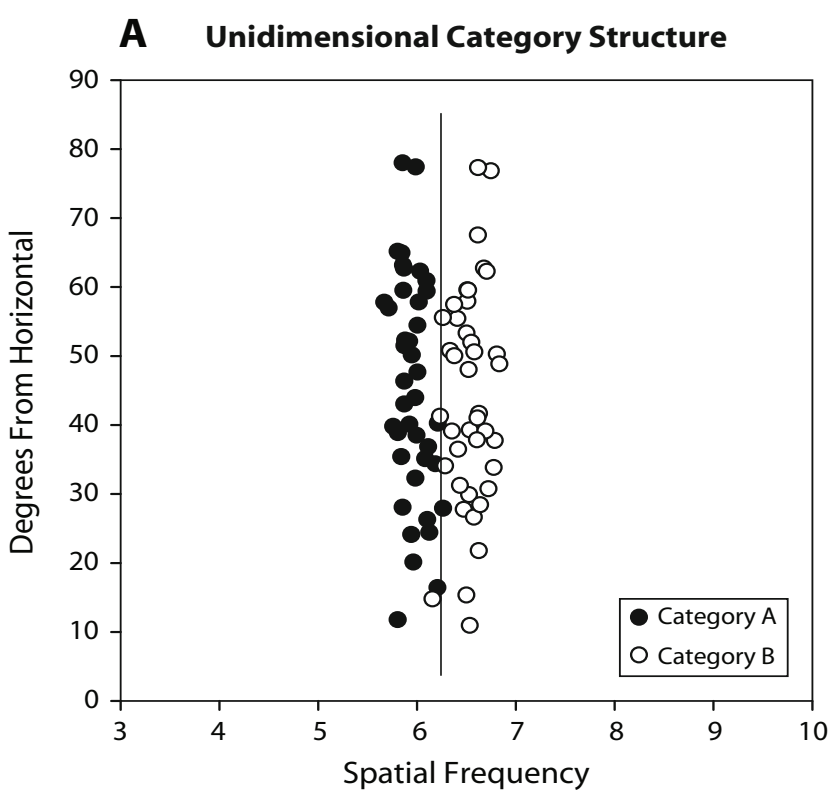

B Diagonal Category Structure

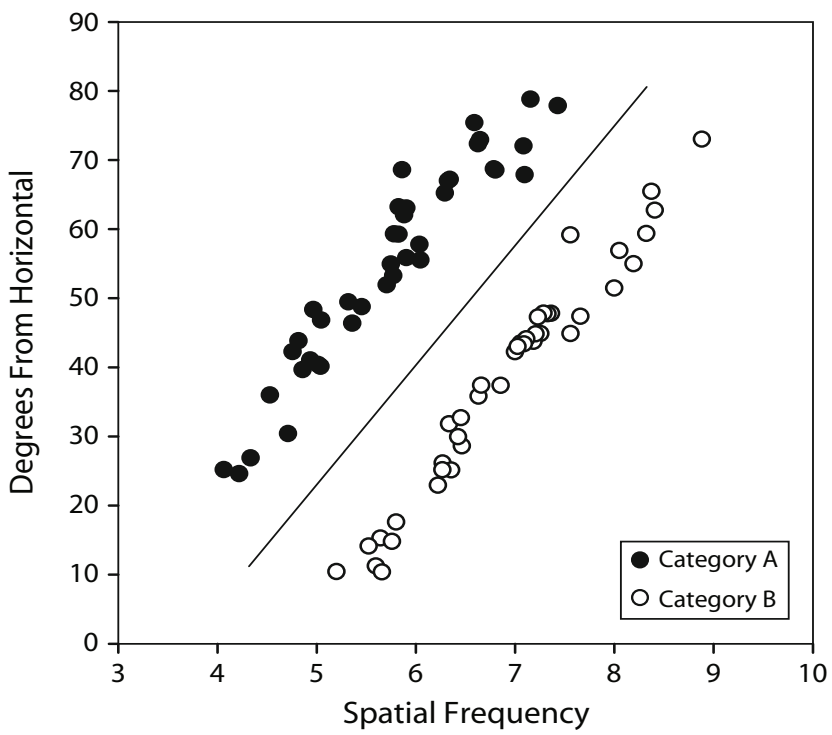

Figure 1. Two category structures and the corresponding optimal decision boundaries for partitioning the space.

An example of an information-integration category structure is Maddox et al.'s (2004) diagonal category structure, depicted in Figure 1B. With respect to information-integration categorization problems, accuracy is maximized only if perceptual information from at least two dimensions is combined prior to the classification decision (Ashby \& Gott, 1988). The diagonal line represents the optimal decision bound for this categorization problem. Importantly, the optimal boundary for an information-integration categorization problem is difficult or impossible to verbalize. In the Figure 1B example, one might describe the boundary as follows: "Respond $A$ if the angle of the bars exceeds their width; otherwise respond B." However, because angle and bar width are not directly comparable, this description is difficult to implement psychologically.

COVIS assumes that rule-based categories, such as the unidimensional structure depicted in Figure 1A, are learned by the explicit system. According to the COVIS processes outlined by Ashby et al. (1998), following feedback that the last response was incorrect, an observer first lowers the strength of the current rule. If the strength of the current rule is below some threshold, the observer selects a new rule and then switches attention to the new rule. These processes require working memory, effort, and importantly, time. In contrast, learning of informationintegration categories is mediated by the implicit system, which processes feedback automatically and does not need access to working memory and attention.

Due to the explicit system's reliance on working memory, Maddox et al. (2004) reasoned that a task that engages working memory immediately after an observer has received feedback would disrupt the observer's ability to process the feedback. Furthermore, by interfering with feedback processing, the task would prevent the observer from engaging in the necessary rule-updating steps required to learn a rulebased category. By contrast, such a task should not disrupt the learning of an information-integration category.

To test this hypothesis, Maddox et al. (2004) conducted experiments in which participants learned either the unidimensional category structure depicted in Figure 1A or the diagonal category structure depicted in Figure 1B. On each trial, after the observer had generated a categorization response and received feedback, he or she engaged in a memory-scanning task. In the memory-scanning task, a set of four digits was displayed for memorization. After a brief delay, a single digit probe was displayed, and the observer's task was to accept or reject the probe as belonging to the memory set. For some participants, the memoryscanning task occurred $500 \mathrm{msec}$ after they had received feedback, whereas other participants received a longer, 2,500-msec delay before the memory-scanning task; these participants had more time to process the feedback. (See Figure 2 for a schematic representation of both the immediate memory-scanning and delayed memory-scanning trials.) If learning of rule-based categories is indeed mediated by an explicit system that needs access to working memory immediately after receiving feedback, then the immediate memory-scanning task should interfere with the observer's ability to learn the unidimensional categories. By contrast, if information-integration categories are learned by a separate implicit procedural learning system, then the immediate memory-scanning task should not interfere with learning in the diagonal categorization problem.

The main result of the Maddox et al. (2004) study was an observed interference in only the immediate memoryscanning task condition of the unidimensional categorization problem. That is, unidimensional classification performance was worse when participants engaged in a memory-scanning task immediately after receiving feedback. However, performance in the diagonal categorization problem was equivalent across the immediate and delayed memory-scanning conditions. Maddox et al. argued that these results supported the claim that two separate 


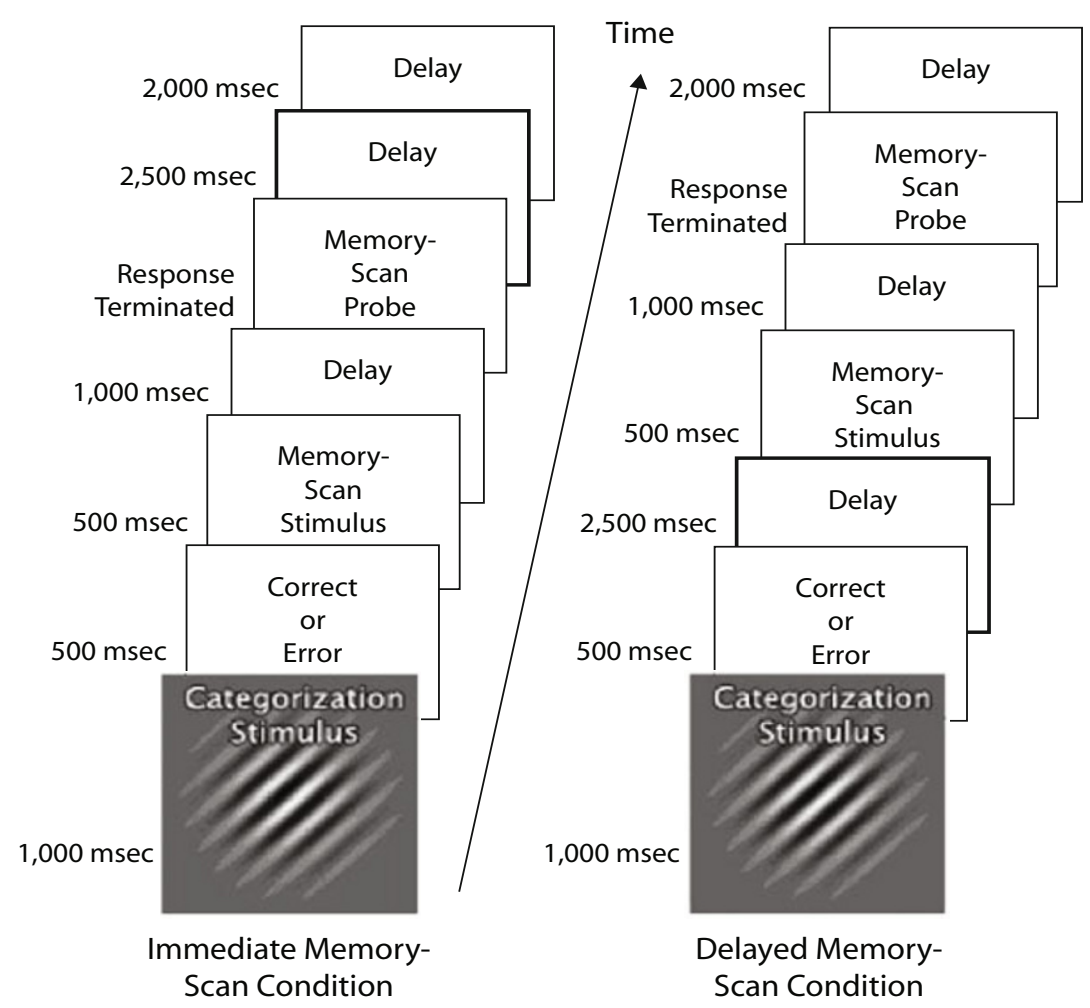

Figure 2. A schematic illustration of the trial sequences in the immediate and delayed memory-scan conditions. (Adapted from Figure 1 of "Disrupting Feedback Processing Interferes With Rule-Based but Not Information-Integration Category Learning," by W. T. Maddox, F. G.Ashby, A. D. Ing, and A. D. Pickering, 2004, Memory \& Cognition, 32, p. 584. Copyright 2004 by the Psychonomic Society, Inc. Adapted with permission.)

systems are responsible for category learning - an explicit system that uses working memory and attentional processes to learn rule-based categories and an implicit system that employs procedural learning to learn informationintegration categories.

The pattern of interference observed in the Maddox et al. (2004) study is intriguing, but we believe that alternative explanations exist that are compatible with a singlesystem approach. In an attempt to equate the difficulty of the rule-based and information-integration categorization problems, Maddox et al. used lower physical spacing between category distributions in the unidimensional category structure, along the relevant dimension, than in the information-integration category structure (compare Figures $1 \mathrm{~A}$ and $1 \mathrm{~B}$ ). The result is that the between-category perceptual discriminability is lower in the unidimensional category structure than in the diagonal category structure. Although Maddox et al.'s attempt to equate the overall learning difficulty of the category structures was well motivated, it is nevertheless the case that the variable of category structure is confounded with the between-category perceptual discriminability of the stimuli.

The goal of the present research is to investigate the role that perceptual discriminability may have played in the Maddox et al. (2004) study. Although we will not propose a detailed processing model, our general line of reasoning is as follows. First, a reasonable assumption is that, early in processing, the visual representation of the classification stimulus is noisy, and that the amount of noise is reduced as processing continues. Furthermore, the introduction of the visual memory-scanning task is likely to prevent continued visual processing and consolidation of the to-be-classified stimulus. We argue that, regardless of the category structure, the feedback must be combined with the visual representation of the to-be-classified stimulus in order for classification learning to occur. Thus, given our visual-noise assumptions, the feedback is likely to be combined with an impoverished visual representation of the stimulus in the immediate memory-scanning condition. The consequence of this process is likely to be particularly detrimental in a case in which between-category discriminability is low to begin with. For example, on some trials, feedback may be combined with a noisy visual representation that overlaps into the incorrect category region. For some observers in the immediate memory-scanning condition, it may never become apparent that there is even a distinction between the categories along the relevant dimension when the between-category discriminability is extremely low. Thus, our central aim in the present research is to decouple category structure type (rule based vs. information integration) from between-category perceptual discriminability to determine the true locus of the interference effects.

To test the perceptual discriminability hypothesis, we follow two approaches. First, we investigate a new rule- 
based category structure with good between-category perceptual discriminability. Furthermore, we attempt to roughly equate the rule-based and information-integration category structures with respect to learning difficulty. That is, our aim is to use a rule-based task that produces an accuracy rate roughly equivalent to the one for the diagonal category structure. If the interference observed by Maddox et al. (2004) was due primarily to the low perceptual discriminability of the stimuli used in their unidimensional categorization, then such interference should not be observed if the discriminability between category distributions is roughly constant across the rule-based and information-integration structures. In the second approach, we test an alternative information-integration structure with lower between-category discriminability than was tested by Maddox et al. If immediate memory scanning results in interference, it would provide convincing evidence that the locus of the effects is in perceptual discriminability and not a reflection of separate learning systems.

\section{EXPERIMENT 1}

In order to ensure correspondence between our study and the Maddox et al. (2004) study, our initial experiment sought to replicate their results. Our replication - in addition to being important so we can verify that we produce the same interference observed by Maddox et al.- -allows us to compare, under identical experimental settings, the results of the unidimensional and diagonal category structures with the alternative category structures planned for this research. In this experiment, we followed the exact procedure used by Maddox et al.

\section{Method}

\section{Participants}

A total of 89 observers from the Indiana University community participated in this experiment. There were 23 and 22 participants in the immediate and delayed memory-scanning conditions of the unidimensional categorization problem, respectively. Twenty-two observers participated in both the immediate and the delayed memoryscanning conditions of the diagonal categorization problem. Of the 89 participants, 31 were paid $\$ 8$ for their participation, and the rest received course credit. A roughly equal number of paid participants were in each of the experimental conditions. Furthermore, all of the participants were informed that they could earn a $\$ 4$ bonus for good performance in the task. They were instructed that to earn the bonus, they needed to achieve at least $70 \%$ accuracy in the categorization task and $90 \%$ accuracy in the memory-scanning task.

\section{Stimuli}

Category structures. There were 40 stimuli that defined each of Categories A and B for both the unidimensional and the diagonal category structures displayed in Figures 1A and 1B. The stimuli were sine wave gratings, and the procedure used for generating the category distributions was the same as that used by Maddox et al. (2004). The stimuli were sampled randomly from bivariate normal distributions defined by the parameters listed in Table 1. Each point $\left(x_{1}, x_{2}\right)$ in the distribution was converted to a stimulus by converting the frequency $\left[f=.25+\left(x_{1} / 50\right)\right]$ and angle $\left[a=x_{2}(\pi / 500)\right]$. Following Maddox et al., a between-category $d^{\prime}$ value can be calculated by computing, along the relevant direction of discrimination, the distance between the means of the category distributions divided by their common standard deviation. As indicated in Table $1, d^{\prime}=$
3.5 for the unidimensional categorization and $d^{\prime}=9.2$ for the diagonal categorization. In independent psychophysical scaling studies, we verified that between-category perceptual discriminability was indeed far worse for the unidimensional categorization than for the diagonal one.

The gratings appeared on a gray background and were displayed on a 15 -in. monitor with $1,024 \times 768$ resolution. Routines supplied by Brainard's (1997) Psychophysics Toolbox were used to generate each of the gratings.

Memory scanning. Four digits were sampled randomly over the range of $0-9$, and no digit was repeated within the set of four. The set of four digits was displayed simultaneously on the center of the screen in 48-point black font set against a gray background, and remained on the screen for $500 \mathrm{msec}$. On half of the trials, the randomly selected single-digit probe came from the memory set, and on half of the trials it did not. The probe was centered on the screen, with the sentence "Was this item in the memory set?" appearing below it.

\section{Procedure}

Category structure and memory-scanning condition were between-subjects variables in the experimental design. The trial sequences for the immediate and delayed memory-scanning conditions are depicted in Figure 2. The sequences are such that the two conditions have equal-length trials.

There were four blocks of trials, and all 80 categorization stimuli were presented in random order within each block. On each trial, a grating was presented for $1,000 \mathrm{msec}$, and then a gray screen was displayed. The participant classified the stimulus into either Category A or Category B by pressing keyboard keys labeled $A$ and $B$. The participant next received $500 \mathrm{msec}$ of feedback. If the participant was in the immediate memory-scanning condition, a gray screen was displayed for $500 \mathrm{msec}$, and then the memory set appeared on the screen for $500 \mathrm{msec}$. In the delayed memory-scanning condition, there was a 2,500-msec pause before the memory set appeared. Following presentation of a gray screen for $1,000 \mathrm{msec}$, the probe digit was presented. The participant then responded whether the probe was or was not a member of the memory set by pressing a key labeled either yes or no. The participants did not receive corrective feedback with regard to the probe item; however, their response, either "yes" or "no," was presented on the screen for $500 \mathrm{msec}$. This was followed by a $4,500-\mathrm{msec}$ or a 2,000 -msec gray screen in the immediate and delayed conditions, respectively.

The participants were instructed that they would engage in a categorization task and a memory task simultaneously. To increase motivation, the participants were informed that they could earn a $\$ 4$ bonus if they achieved $70 \%$ accuracy on the categorization portion and $90 \%$ accuracy on the memory portion. Furthermore, the dimensions of the gratings were explained, and the participants were instructed that "In some conditions it may be difficult to determine the values of a dimension; however, you will need to do so to learn to categorize the stimuli and earn the $\$ 4$ bonus."

Table 1

Population Parameter Values Used to Generate the Stimuli in Experiment 1

\begin{tabular}{lccccr}
\hline \multicolumn{1}{c}{ Distributions } & $\mu_{\mathrm{F}}$ & $\mu_{\mathrm{A}}$ & $\sigma_{\mathrm{F}}^{2}$ & $\sigma_{\mathrm{A}}^{2}$ & $\sigma_{\mathrm{FA}}$ \\
\hline Unidimensional $\left(d^{\prime}=3.5\right)$ & & & & & \\
$\quad$ Category A & 285 & 125 & 75 & 2,000 & 0 \\
$\quad$ Category B & 315 & 125 & 75 & 2,000 & 0 \\
Diagonal $\left(d^{\prime}=9.2\right)$ & & & & & \\
$\quad$ Category A & 272 & 153 & 4,000 & 4,000 & 3,934 \\
$\quad$ Category B & 327 & 97 & 4,000 & 4,000 & 3,934 \\
\hline
\end{tabular}

Note- $\mu_{\mathrm{F}}$, mean frequency; $\mu_{\mathrm{A}}$, mean angle; $\sigma_{\mathrm{F}}^{2}$, variance frequency; $\sigma_{\mathrm{A}}^{2}$, variance angle; $\sigma_{\mathrm{FA}}$, covariance length-angle. The frequency is measured in cycles per degree, and the angle values are measured in radians. 


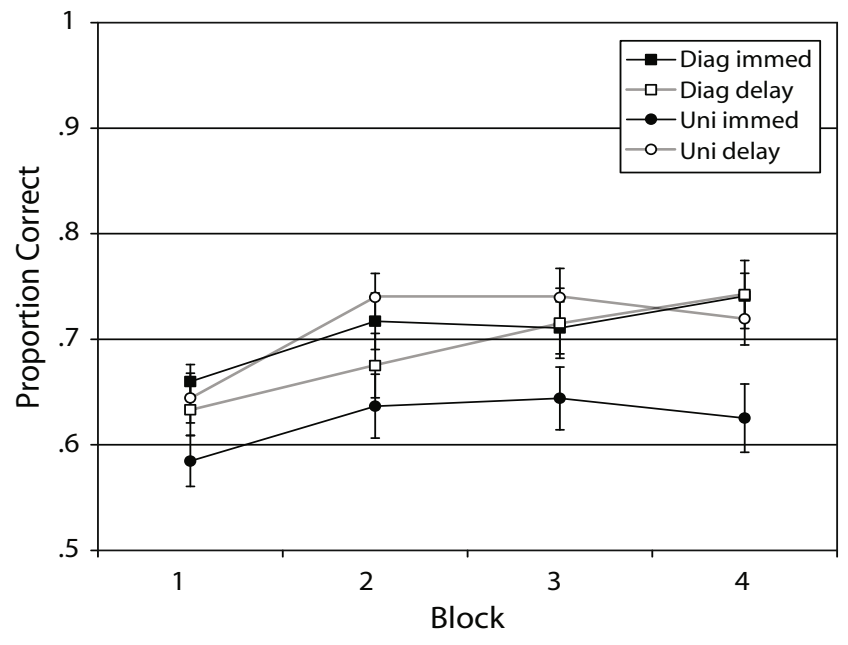

Figure 3. The mean probability of correct classifications as a function of category structure (unidimensional or diagonal), condition (immediate or delayed), and block in Experiment 1.

\section{Results}

Overall memory-scanning accuracy averaged $97.1 \%$ correct across the different conditions. There was no main effect of condition (immediate or delay) in either the unidimensional categorization task $[F(1,42)=1.065$, $\left.M S_{\mathrm{e}}=.021, p=.308\right]$ or the diagonal categorization task $\left[F(1,43)=0.450, M S_{\mathrm{e}}=.003, p=.506\right]$. Furthermore, there was no main effect of category type (unidimensional or diagonal) $\left[F(1,85)=1.375, M S_{\mathrm{e}}=.011, p=.244\right]$ and no condition $\times$ category type interaction $[F(1,85)=$ $\left.1.455, M S_{\mathrm{e}}=.011, p=.231\right]$.

The mean proportion of correct classifications is shown as a function of category type, condition, and block (1-4) in Figure 3. The pattern of results depicted in Figure 3 closely mirrors that observed by Maddox et al. (2004). The key finding is that immediate memory scanning interferes with performance in the unidimensional categorization, but not in the diagonal categorization. A 2 (category type) $\times 2$ (condition) $\times 4$ (block) ANOVA was conducted on the accuracy data. The main effect of block $[F(3,255)=25.916$, $\left.M S_{\mathrm{e}}=.004, p<.001\right]$ was significant, indicating that accuracy was improving across the blocks. The main effects of category type $\left[F(1,85)=1.837, M S_{\mathrm{e}}=.052, p=.179\right]$ and condition $\left[F(1,85)=2.259, M S_{\mathrm{e}}=.052, p=.137\right]$ were not significant; however, the category type $\times$ condition interaction was significant $\left[F(1,85)=4.651, M S_{\mathrm{e}}=\right.$ $.052, p=.034]$, confirming the key observation noted above. To examine the results more closely, we performed additional analyses separately for the unidimensional and diagonal category types.

For the unidimensional category data, the main effect of block was again significant $[F(3,126)=12.064$, $\left.M S_{\mathrm{e}}=.005, p<.001\right]$, reflecting improvement in accuracy from early blocks to later blocks. In contrast to the analyses above examining the main effect of condition across both category types, the main effect of condition for the unidimensional category data was significant
$\left[F(1,42)=6.925, M S_{\mathrm{e}}=.049, p=.012\right]$, confirming the interference produced by immediate memory scanning. The block $\times$ condition interaction $[F(3,126)=$ $\left.0.881, M S_{\mathrm{e}}=.005, p=.453\right]$ was not significant. For the diagonal category data, the main effect of block was significant $\left[F(3,129)=17.902, M S_{\mathrm{e}}=.004, p<.001\right]$, but the main effect of condition $\left[F(1,43)=0.207, M S_{\mathrm{e}}=\right.$ $.054, p=.651]$ and the interaction of block $\times$ condition $\left[F(3,129)=1.422, M S_{\mathrm{e}}=.004, p=.239\right]$ were both nonsignificant.

As an additional analysis of the accuracy performance, we examined the proportion of observers who learned the category structure. In accord with analyses performed by Maddox et al. (2004), we defined learners as those participants with a mean proportion correct of at least .65 within any window of 80 trials. That is, the mean proportion correct for Trials 1-80 was first computed. If this result was below .65, then the mean proportion correct for Trials 2-81 was computed, and so on, until a window of 80 trials with mean proportion correct greater than .65 was observed. Participants who met the criterion were classified as learners, and those who did not were classified as nonlearners.

The proportion of learners for each condition is displayed in Figure 4. Chi-squared tests were performed on the proportions of learners and nonlearners in the immediate and delay conditions of the unidimensional categorization task, and separately for the immediate and delay conditions of the diagonal categorization task. A significantly greater proportion of participants failed to learn the unidimensional category structure in the immediate memory-scanning condition than in the delayed memoryscanning condition $\left[\chi^{2}(1)=8.844, p=.003\right]$. For the diagonal categorization task, there was not a significant difference between the proportion of learners in the immediate and delayed memory-scanning conditions $\left[\chi^{2}(1)=\right.$ $0.407, p=.524]$.

\section{Discussion}

The results of Experiment 1 replicate those observed by Maddox et al. (2004). Immediate memory scanning led to interference in the unidimensional categorization, but not in the diagonal categorization. According to the Maddox et al. hypothesis, the immediate memory-scanning task should interfere with the explicit system's ability to process feedback and undergo rule-updating procedures. Thus, these researchers interpreted the observed poor performance in the immediate memory-scanning condition of the unidimensional categorization task as evidence supporting this hypothesis. Additionally, according to Maddox et al., the lack of any interference in the diagonal categorization task was further evidence that the learning of information-integration categories relies on an implicit system that does not make use of working memory and attentional processes. Having verified the Maddox et al. results, we now pursue our investigation of the alternative idea that these results are due to an imbalance of perceptual discriminability between the unidimensional and the diagonal category structures. 


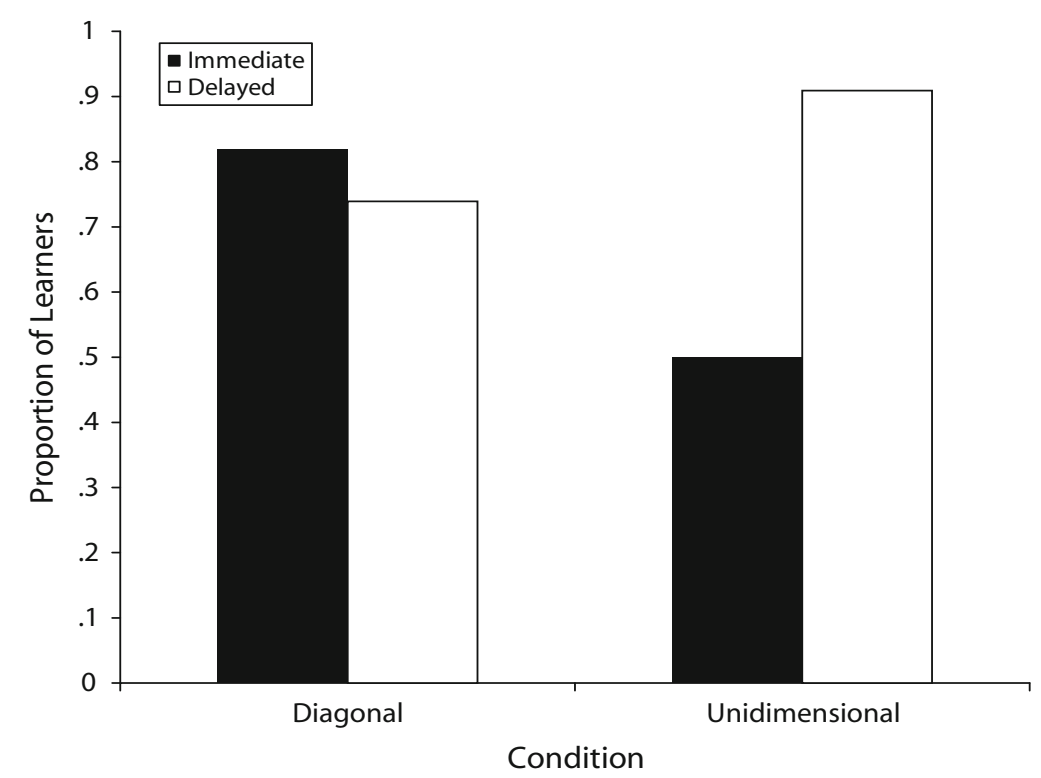

Figure 4. Proportion of observers who met the learning criterion as a function of category structure (unidimensional or diagonal) and condition (immediate or delayed) in Experiment 1.

\section{EXPERIMENT 2}

In this experiment, we attempt to demonstrate that the immediate memory-scanning task does not produce interference when observers learn an alternative rule-based category structure with good between-category perceptual discriminability. Such a demonstration is interesting, however, only to the extent that the overall accuracy rate is roughly equivalent to what was observed for the previously tested unidimensional and diagonal category structures. If the categorization task is too easy and performance is at ceiling, then task difficulty effects could explain a failure to observe interference caused by the memory-scanning task. Likewise, if the task is too difficult and performance is at floor, task difficulty effects could again explain the lack of an observed interference.

To achieve the desired goals, we again tested a rulebased structure in which a single dimension was relevant. However, rather than introducing difficulty by using hard-to-discriminate stimuli, we introduced difficulty by increasing the number of irrelevant dimensions that composed the objects. Furthermore, the between-category discriminability along the single relevant dimension was approximately the same as for the diagonal task tested by Maddox et al. (2004) and in our Experiment 1.

Specifically, the stimuli in this experiment are sine wave gratings that are enclosed by a rectangle that varies in height. Also enclosed in the rectangle is a vertical line that varies in horizontal placement. The stimuli have four dimensions, yet for any given participant, only a single dimension determined category membership. (We refer to the Experiment 2 category structure as the 4-D structure, to avoid confusing this unidimensional category structure with the unidimensional category structure used in Experiment 1.)
A sample category structure is depicted in Figure 5. Although there are four possible single-dimension rules for this stimulus set, in this example, only the height of the rectangle determines category membership (the horizontal line in Figure 5 represents the optimal decision boundary); the other three dimensions are irrelevant. The simple verbal rule for correct classification corresponds to: "Members of Category A have short rectangles and members of Category B have tall rectangles."

Pilot work suggested that learning performance on the 4-D structure would be in roughly the same range as on the unidimensional and diagonal tasks from Experiment 1 and so would not suffer from either ceiling or floor effects. Critically, however, the between-category $d^{\prime}$ of this rule-based 4-D structure ( $d^{\prime}=11.0$ or 7.8 , depending on the relevant dimension) is higher than that of the unidimensional structure $\left(d^{\prime}=3.5\right)$ and of roughly the same magnitude as that of the diagonal structure $\left(d^{\prime}=9.2\right)$ of Experiment 1. Furthermore, independent psychophysical scaling studies indicated good between-category perceptual discriminability, regardless of which dimension defined the categories. This scaling work indicated that the between-category perceptual discriminability for the 4-D structure was about the same as for the diagonal structure from Experiment 1. Therefore, our hypothesis is that the immediate memory-scanning task will not impair learning in the 4-D structure.

\section{Method}

\section{Participants}

A total of 96 observers, drawn from the same population as in Experiment 1, participated in this experiment. There were 47 participants in the immediate memory-scanning condition and 49 in the delayed memory-scanning condition. Nineteen of the participants in the immediate memory-scanning condition and 18 of the 

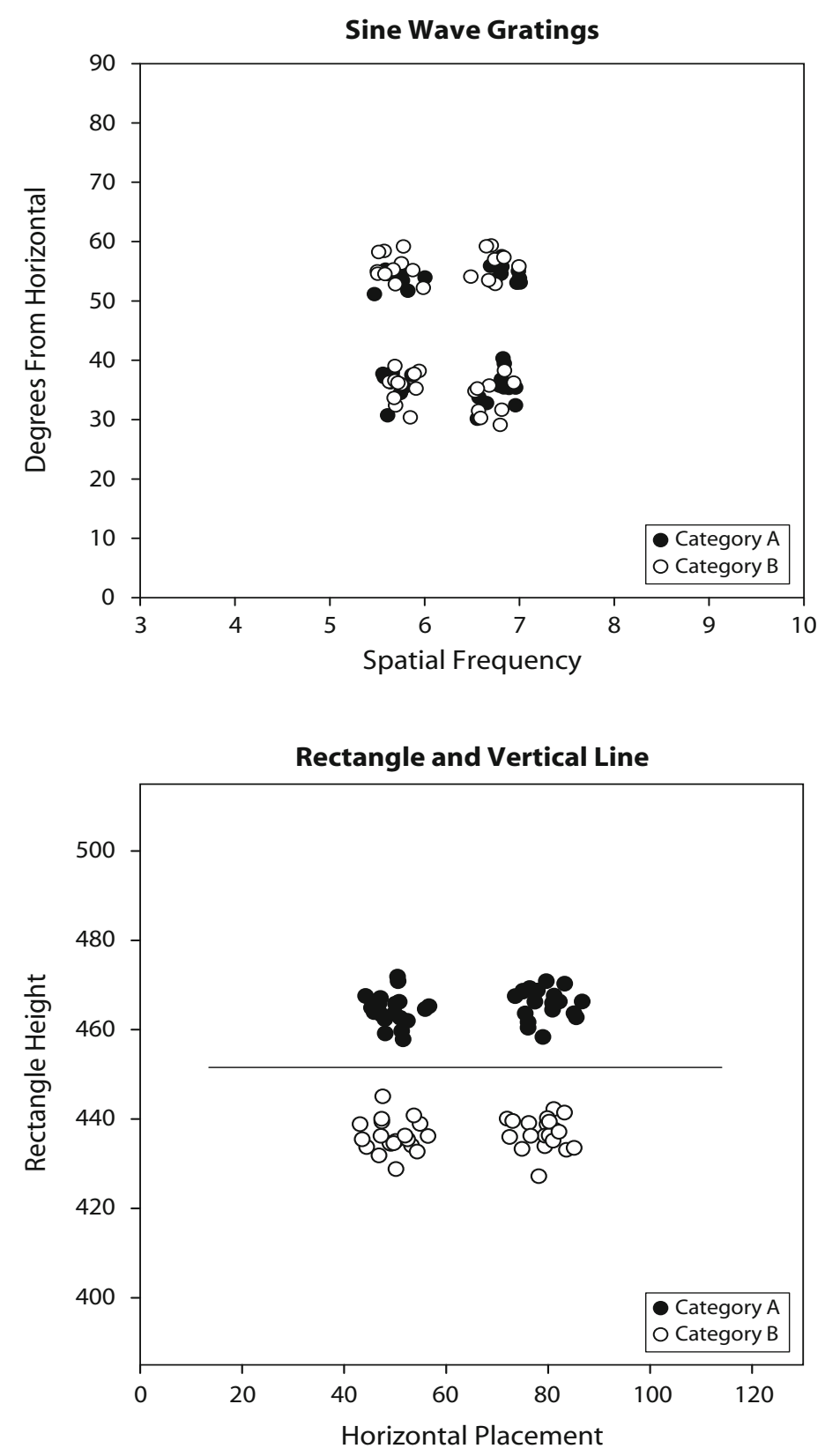

Figure 5. Experiment 2: An example of the 4-D category structure and its corresponding optimal decision boundary.

participants in the delayed memory-scanning condition received payment for their participation. Note that we doubled the number of participants in these conditions, relative to the original experiments conducted by Maddox et al. (2004), to address any concerns regarding statistical power.

\section{Stimuli}

The categorization stimuli were sine wave gratings varying in spatial frequency and angle. Adjacent to the grating was a vertical line that varied in horizontal placement, and both the grating and the vertical line were enclosed by a rectangle that varied in height. An example of one of the experimental stimuli is shown in Figure 6 . The parameters used to generate the category distributions are listed in Tables 2 and 3, and an example of a resulting category structure is depicted in Figure 5. The assignment of which single dimension was the relevant rule dimension was chosen randomly for each participant.

\section{Procedure}

To ensure that the observers understood the stimulus configuration, we displayed an example stimulus on the screen before the experiment and described it as follows: "In the categorization task, each stimulus varies along four dimensions. An example of a categorization stimulus is below. The four dimensions that vary are the height of the rectangle, the left-to-right placement of the enclosed vertical line, the angle of the lines in the fuzzy patch, and the width of the lines in the fuzzy patch." Other than the altered instructions, all other aspects of the procedure were identical to those of the previous experiment. 


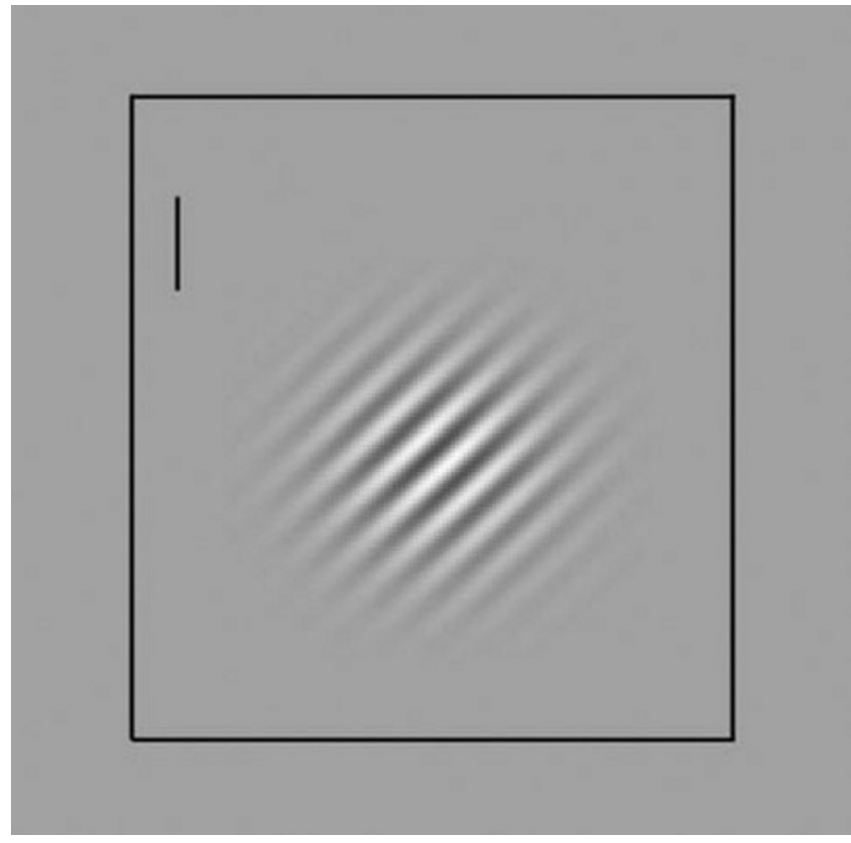

Figure 6. An example of one of the stimuli in Experiment 2.

\section{Results}

Overall memory-scanning accuracy averaged $97.2 \%$ correct. There was no main effect of condition (immediate vs. delayed) on memory-scanning accuracy $[F(1,94)=$ $\left.0.001, M S_{\mathrm{e}}=.010, p=.977\right]$.

The mean proportion of correct classifications for the immediate and delayed memory-scanning conditions is displayed as a function of block in Figure 7. In contrast to the results from the unidimensional structure of Experiment 1 , the categorization accuracy in the 4-D category structure is not significantly different across the two levels of condition $\left[F(1,94)<0.001, M S_{\mathrm{e}}=.141, p=.996\right]$. The main effect of block was significant $[F(3,94)<52.166$, $\left.M S_{\mathrm{e}}=.467, p<.001\right]$; however, the block $\times$ condition interaction was not significant $\left[F(3,94)<1.010, M S_{\mathrm{e}}=\right.$ $.009, p=.388]$. More detailed analyses revealed that there was no main effect of which dimension served as the rule dimension for the 4-D structure $[F(3,88)=1.453$, $\left.M S_{\mathrm{e}}=.131, p=.233\right]$. Also, there was no dimension $\times$ condition (immediate vs. delayed) interaction $[F(3,88)=$ $\left.0.182, M S_{\mathrm{e}}=.137, p=.908\right]$.

To further compare the accuracy performance across the two levels of condition in the 4-D structure, we analyzed the proportion of observers who reached the learning criterion, as outlined in Experiment 1. The proportions of observers who met the learning criterion in the immediate and delayed memory-scanning conditions was .72 and .74 , respectively. There was not a significant difference in the proportion of learners across the two conditions $\left[\chi^{2}(1)=0.062, p=.803\right]$.

\section{Discussion}

The results from Experiment 2 support the hypothesis that the interference observed by Maddox et al. (2004),
Table 2

Population Parameter Values Used to Generate the Stimuli in Experiment 2, Sine Wave Gratings $\left(d^{\prime}=11.0\right)$

\begin{tabular}{lcrccc}
\hline Distributions & $\mu_{\mathrm{F}}$ & $\mu_{\mathrm{A}}$ & $\sigma_{\mathrm{F}}^{2}$ & $\sigma_{\mathrm{A}}^{2}$ & $\sigma_{\mathrm{FA}}$ \\
\hline Category A1 & 272 & 153 & 25 & 25 & 0 \\
Category A2 & 327 & 97 & 25 & 25 & 0 \\
Category B1 & 327 & 153 & 25 & 25 & 0 \\
Category B2 & 272 & 97 & 25 & 25 & 0 \\
\hline
\end{tabular}

Note- $-\mu_{\mathrm{F}}$, mean frequency; $\mu_{\mathrm{A}}$, mean angle; $\sigma_{\mathrm{F}}^{2}$, variance frequency; $\sigma_{\mathrm{A}}^{2}$, variance angle; $\sigma_{\mathrm{FA}}$, covariance length-angle. The frequency is measured in cycles per degree, and the angle values are measured in radians.

and again in our Experiment 1, was due primarily to poor perceptual discriminability interacting with the immediate memory-scanning task. Like the unidimensional structure tested in Experiment 1, the 4-D structure is a rulebased task in which only a single dimension is relevant to classification. Although the 4-D structure is a rule-based task, the immediate memory-scanning task failed to induce greater interference than did the delayed memoryscanning task.

We suspect that, given a sufficiently sensitive experimental design and an appropriate choice of experimental parameters, manipulations that disrupt the processing of feedback could be shown to produce interference regardless of the category structure. Nevertheless, the finding of no interference in the present experiment is important because, except for the between-category discriminability of the stimuli, it was observed under conditions that were essentially the same as those for the unidimensional task tested by Maddox et al. (2004). Thus, the results suggest that perceptual discriminability, rather than the type of category structure (rule based vs. information integration), was the primary mediator of the feedback-interference effects from Maddox et al.'s original study.

\section{EXPERIMENT 3}

To the extent that the rule-based interference observed in Experiment 1 was due to poor perceptual discriminability, immediate memory scanning should also induce interference in an information-integration category structure with sufficiently poor perceptual discriminability. The purpose of this experiment was to demonstrate that when the between-category perceptual discriminability of an information-integration category structure is poor, the

Table 3

Population Parameter Values Used to Generate the Stimuli in Experiment 2, Rectangle and Line $\left(d^{\prime}=7.8\right)$

\begin{tabular}{cccccc}
\hline Distributions & $\mu_{\mathrm{H}}$ & $\mu_{\mathrm{P}}$ & $\sigma_{\mathrm{H}}^{2}$ & $\sigma_{\mathrm{P}}^{2}$ & $\sigma_{\mathrm{HP}}$ \\
\hline Category A1 & 465 & 50 & 15 & 15 & 0 \\
Category A2 & 465 & 80 & 15 & 15 & 0 \\
Category B1 & 435 & 50 & 15 & 15 & 0 \\
Category B2 & 435 & 80 & 15 & 15 & 0 \\
\hline
\end{tabular}

Note- $\mu_{\mathrm{H}}$, mean height difference; $\mu_{\mathrm{P}}$, mean placement difference; $\sigma_{\mathrm{H}}^{2}$, variance height; $\sigma_{\mathrm{P}}^{2}$, variance placement; $\sigma_{\mathrm{HP}}$, covariance heightplacement. The height of the rectangle is measured in pixels, and the placement is measured in pixel distance from the left edge of the rectangle. 


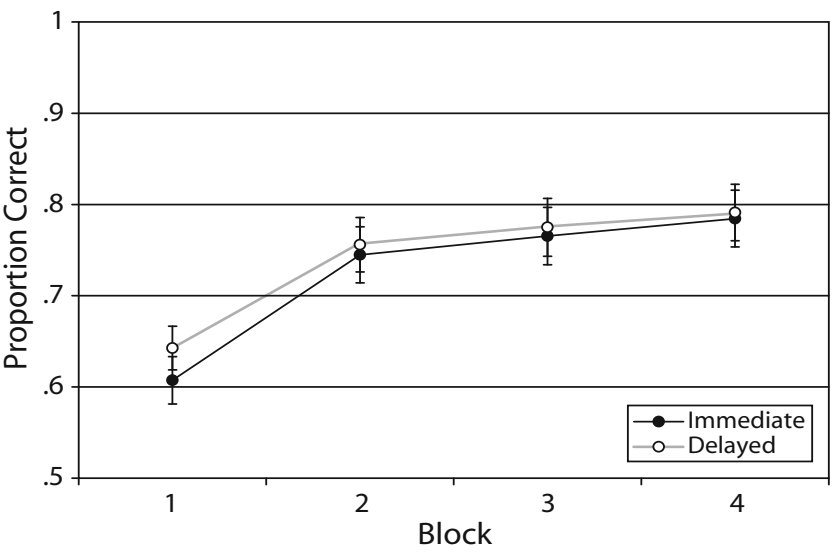

Figure 7. The mean probability of correct classifications as a function of condition (immediate or delayed) and block in Experiment 2.

immediate memory-scanning task will yield worse performance than the delayed memory-scanning task. Such a demonstration would provide convincing evidence that perceptual discriminability, rather than the type of category structure (rule based vs. information integration), is the key factor mediating the interference effects.

\section{Method}

\section{Participants}

A total of 32 participants, drawn from the same population as in Experiments 1 and 2, participated in this experiment. There were equal numbers of participants in the immediate and delayed memory-scanning conditions. All of the participants in this experiment were paid for their participation.

\section{Stimuli}

The information-integration category structure that we used is illustrated in Figure 8. It is similar to the diagonal category structure in Experiment 1; however, the mean separation distance between the two category distributions is smaller (resulting $d^{\prime}=4.8$ ). We will refer to the modified structure as the low- $d^{\prime}$ diagonal category structure. Again, the categorization stimuli were sine wave gratings varying in spatial frequency and angle. We generated the structure by sampling from two bivariate normal distributions. The parameters used to generate these distributions are listed in Table 4. Along both dimensions, the difference between the population means was set at 30 units. These values were chosen in an attempt to decrease the between-category discriminability of the diagonal structure, but to maintain the possibility of reasonable learning performance. An independent psychophysical scaling study indicated that betweencategory perceptual discriminability was indeed significantly worse for the low- $d^{\prime}$ diagonal structure than for the diagonal structure tested

Table 4

Population Parameter Values Used to Generate the Stimuli in Experiment 3, Low- $d^{\prime}$ Diagonal $\left(d^{\prime}=4.8\right)$

\begin{tabular}{lccccc}
\hline Distributions & $\mu_{\mathrm{F}}$ & $\mu_{\mathrm{A}}$ & $\sigma_{\mathrm{F}}^{2}$ & $\sigma_{\mathrm{A}}^{2}$ & $\sigma_{\mathrm{FA}}$ \\
\hline Category A & 285 & 140 & 4,000 & 4,000 & 3,934 \\
Category B & 315 & 110 & 4,000 & 4,000 & 3,934 \\
\hline
\end{tabular}

Note $-\mu_{\mathrm{F}}$, mean frequency; $\mu_{\mathrm{A}}$, mean angle; $\sigma_{\mathrm{F}}^{2}$, variance frequency; $\sigma_{\mathrm{A}}^{2}$, variance angle; $\sigma_{\mathrm{FA}}$, covariance length-angle. The frequency is measured in cycles per degree, and the angle values are measured in radians. in our Experiment 1. The construction of the memory-scanning stimuli was identical to that described in Experiment 1.

\section{Procedure}

The experimental procedure was identical to that used in Experiment 1.

\section{Results}

Memory-scanning performance averaged $98.6 \%$ correct and did not differ significantly across the immediate and delayed conditions $\left[F(1,30)=0.115, M S_{\mathrm{e}}=.001\right.$, $p=.737]$.

The mean proportion of correct categorization responses is displayed as a function of condition and block in Figure 9. In contrast to the results of the diagonal category structure in Experiment 1, the categorization accuracy in the immediate memory-scanning condition was significantly lower than that in the delayed memoryscanning condition $\left[F(1,30)=6.283, M S_{\mathrm{e}}=.016, p=\right.$ $.018]$. Furthermore, both the effect of block $[F(1,30)=$ 74.544, $\left.M S_{\mathrm{e}}=.003, p<.001\right]$ and the block $\times$ condition interaction $\left[F(1,30)=4.592, M S_{\mathrm{e}}=.001, p=.04\right]$ were significant.

In accord with the previous experiments, we performed an additional analysis comparing the proportion of learners across the two conditions. To avoid ceiling effects in the proportion of learners, we set the learning criterion for any 80 -trial window at .70 rather than at .65 . The proportion of learners in the immediate and delayed conditions $(.56$ and .88$)$ differed significantly $\left[\chi^{2}(1)=3.87\right.$, $p=.049]$.

\section{Discussion}

In sum, Maddox et al. (2004) derived a prediction from the multiple-system COVIS model that immediate memory scanning should not interfere with the learning of information-integration category structures. The present results contradict this prediction, with learning of the low- $d^{\prime}$ diagonal structure being significantly worse in the immediate memory-scanning condition than in the delayed condition.

Indeed, note that across Experiments 2 and 3 of the present research, we produced a reverse dissociation from the one predicted and observed by Maddox et al. (2004). These researchers predicted that immediate memory scanning would interfere with the learning of rule-based categories but would not interfere with the learning of information-integration categories. They reported data consistent with this predicted dissociation in their original experiments, and we replicated their results in our Experiment 1. However, across our Experiments 2 and 3, we produced the reverse dissociation: Immediate memory scanning interfered with the learning of an informationintegration structure (the low- $d^{\prime}$ diagonal categorization of Experiment 3) but did not interfere with the learning of a rule-based category structure (the 4-D structure of Experiment 2). The common denominator across all of the experiments is that, when between-category perceptual discriminability is low, immediate memory scanning interferes with category learning. The results thereby 


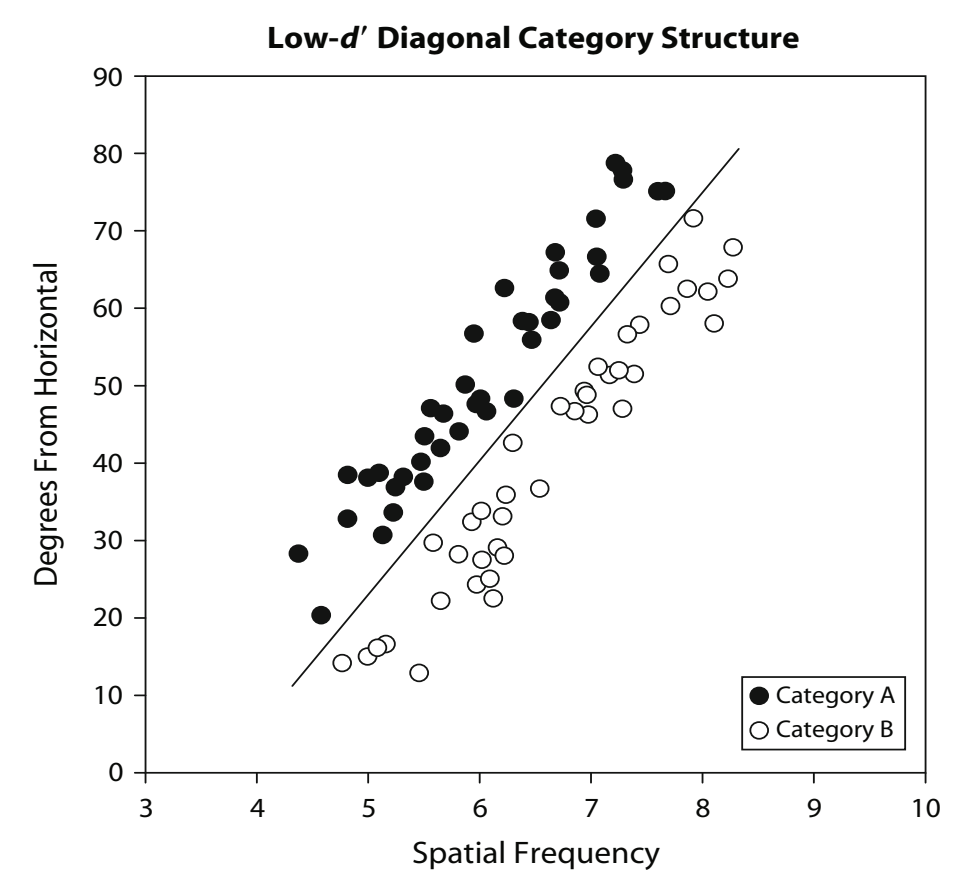

Figure 8. Experiment 3: The low- $d^{\prime}$ diagonal category structure and its corresponding optimal decision boundary.

challenge Maddox et al.'s original interpretation that their observed dissociation was a reflection of separate learning systems for rule-based and information-integration category structures.

\section{GENERAL DISCUSSION}

An important debate in the perceptual classification literature is whether a single system or multiple cognitive systems underlie the representation of categories. Furthermore, in the context of this debate, one of the major issues has involved the interpretation of a variety of dissociations of category learning (Ashby \& Maddox, 2005; Kinder \& Shanks, 2001; Knowlton \& Squire, 1993; Nosofsky \& Kruschke, 2002; Nosofsky \& Zaki, 1998; Palmeri \& Flanery, 1999; Reber, Gitelman, Parrish, \& Mesulam, 2003; Reed, Squire, Patalano, Smith, \& Jonides, 1999; Shanks \& St. John, 1994; Zaki, 2004; Zaki \& Nosofsky, 2001). Of most relevance to the present work, Ashby, Maddox, and their colleagues have demonstrated a long series of dissociations consistent with the predictions from the multiple-systems COVIS framework. According to COVIS, an explicit system is responsible for learning rule-based categories by using working memory to assess the validity of candidate rules. By contrast, an implicit system learns information-integration categories via a form of procedural learning. The motivation for demonstrating dissociations is based on the following logic. If category learning is mediated by a single representational system, then a manipulation that interferes with processing of one category type should also interfere with processing of another category type. If a given experimental manipulation impairs learning of one of the basic category types, whereas learning of the other category type is unaffected, then the result is often taken as evidence for the existence of separate and distinct representational systems. Although Ashby, Maddox, and their colleagues have demonstrated numerous such dissociations with this logic in mind (see Maddox \& Ashby, 2004, for a review), there is growing evidence that plausible alternative explanations exist for many of the observed dissociations.

For example, Ashby, Ell, and Waldron (2003) reported a dissociation involving a procedural manipulation based on switching the mapping of category membership to response buttons. The manipulation interfered with performance on an information-integration category structure

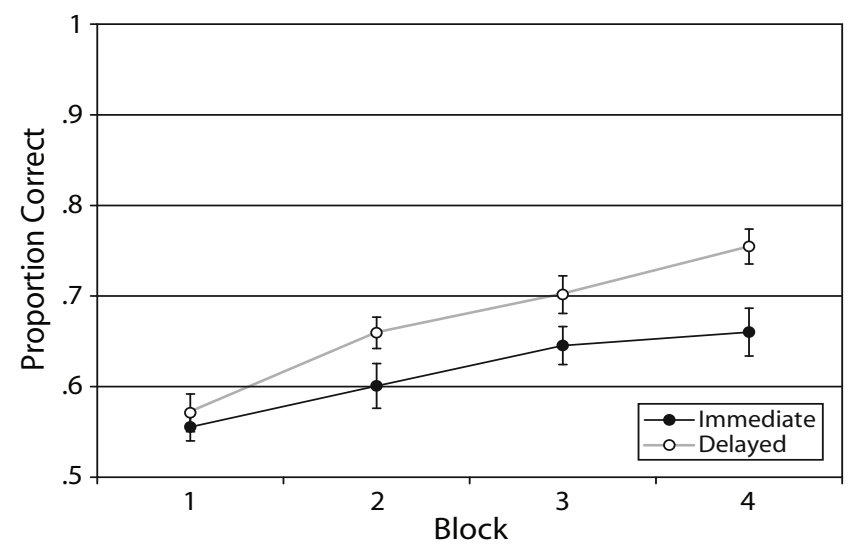

Figure 9. The mean probability of correct classifications as a function of condition (immediate or delayed) and block in Experiment 3. 
but not on a rule-based category structure. This dissociation was consistent with the COVIS assumption that a procedural system mediates the learning of informationintegration categories, whereas a separate explicit system learns rule-based categories. However, as acknowledged by Ashby et al. (2003), the information-integration and rule-based structures were not equated on all forms of "difficulty." Nosofsky, Stanton, and Zaki (2005) provided converging sources of evidence that the dissociation observed by Ashby et al. (2003) was in fact due to the difficulty factor and not to separate systems mediating the learning of rule-based versus information-integration category structures.

In the present research, we have addressed another dissociation that was presented in support of the COVIS model. According to COVIS, the explicit learning system uses working memory and attention to store and assess the validity of verbal rules. Maddox et al. (2004) reasoned that any task that engages working memory immediately after the observer receives corrective feedback should interfere with an observer's ability to learn a rule-based category. However, because learning of information-integration categories is mediated by an implicit system rooted in procedural learning, performance on information-integration structures should be unaffected by the memory task. In support of these predictions, in the Maddox et al. study, a memory-scanning task that occurred shortly after the presentation of corrective feedback interfered with the participants' ability to learn a rule-based structure but not with their ability to learn an information-integration structure.

As noted in the present article, however, in Maddox et al.'s (2004) study, the variable of category structure (rule based vs. information integration) was confounded with between-category perceptual discriminability. Furthermore, we hypothesized that in Maddox et al.'s study, the immediate memory-scanning task interfered with performance only in the rule-based unidimensional condition because of the very low perceptual discriminability of the stimuli that were used. In support of this hypothesis, in the present research, we demonstrated that the immediate memory-scanning task failed to produce any interference for a rule-based category structure involving easy-to-discriminate stimuli. By contrast, the immediate memory-scanning task did interfere with the learning of an information-integration structure with hard-to-discriminate stimuli. In a nutshell, therefore, we were able to produce the reverse dissociation of what was predicted by the COVIS model. The results therefore provide evidence that it is between-category perceptual discriminability, and not the operation of separate rulebased versus information-integration learning systems, that underlies the present dissociation effects.

In sum, although there exist numerous reported dissociations in support of the multiple-systems COVIS framework, we believe that the results of the present research, in combination with those in other studies (Lagnado, Newell, Kahan, \& Shanks, 2006; Nosofsky \& Johansen, 2000; Nosofsky \& Kruschke, 2002; Nosofsky et al., 2005), are beginning to raise important questions about their interpretation. In particular, on the basis of our research thus far, the dissociations seem to disappear when key extraneous experimental factors are controlled (or else are robust predictions of the alternative single-system models themselves).

Unfortunately, it may be extraordinarily difficult to simultaneously control all relevant extraneous factors when comparing performance on rule-based versus information-integration category structures. This problem potentially undermines the research strategy of relying on the demonstration of experimental dissociations as a basis for providing evidence for the operation of the multiple category-learning systems. An alternative research strategy might be to formulate more fully specified versions of both multiple-system and single-system models and to evaluate their ability to account quantitatively for the full spectrum of performance across multiple tasks. The extensive studies reported by Ashby, Maddox, and their colleagues on the learning of rule-based and informationintegration categories provide a fertile testing ground for pursuing such an alternative research strategy.

\section{AUTHOR NOTE}

This work was supported by Grant R01 MH48494 from the National Institute of Mental Health. Correspondence concerning this article should be addressed to R. M. Nosofsky, Department of Psychological and Brain Sciences, Indiana University, Bloomington, IN 47405 (e-mail: nosofsky@indiana.edu).

\section{REFERENCES}

Ashby, F. G., Alfonso-Reese, L. A., Turken, A. U., \& Waldron, E. M. (1998). A neuropsychological theory of multiple systems in category learning. Psychological Review, 105, 442-481.

Ashby, F. G., Ell, S. W., \& Waldron, E. M. (2003). Procedural learning in perceptual categorization. Memory \& Cognition, 31, 1114-1125.

Ashby, F. G., \& GotT, R. E. (1988). Decision rules in the perception and categorization of multidimensional stimuli. Journal of Experimental Psychology: Learning, Memory, \& Cognition, 14, 33-53.

Ashby, F. G., \& Maddox, W. T. (2005). Human category learning. Annual Review of Psychology, 56, 149-178.

Brainard, D. H. (1997). The Psychophysics Toolbox. Spatial Vision, 10, 433-436.

Erickson, M. A., \& KruschKe, J. K. (1998). Rules and exemplars in category learning. Journal of Experimental Psychology: General, 127, 107-140.

Kinder, A., \& Shanks, D. R. (2001). Amnesia and the declarative/ nondeclarative distinction: A recurrent network model of classification, recognition, and repetition priming. Journal of Cognitive Neuroscience, 13, 648-669.

Knowlton, B. J., \& Squire, L. R. (1993). The learning of categories: Parallel brain systems for item memory and category knowledge. Science, 262, 1747-1749.

Lagnado, D. A., Newell, B. R., Kahan, S., \& Shanks, D. R. (2006). Insight and strategy in multiple-cue learning. Journal of Experimental Psychology: General, 135, 162-183.

Maddox, W. T., \& Ashby, F. G. (2004). Dissociating explicit and procedural-learning based systems of perceptual category learning. Behavioral Processes, 66, 309-332.

Maddox, W. T., Ashby, F. G., Ing, A. D., \& Pickering, A. D. (2004). Disrupting feedback processing interferes with rule-based but not information-integration category learning. Memory \& Cognition, 32, 582-591.

Nosofsky, R. M., \& Johansen, M. K. (2000). Exemplar-based accounts of "multiple-system" phenomena in perceptual categorization. Psychonomic Bulletin \& Review, 7, 375-402.

Nosofsky, R. M., \& KruschKe, J. K. (2002). Single-system models and interference in category learning: Commentary on Waldron and Ashby (2001). Psychonomic Bulletin \& Review, 9, 169-174. 
Nosofsky, R. M., Stanton, R. D., \& Zaki, S. R. (2005). Procedural interference in perceptual classification: Implicit learning or cognitive complexity? Memory \& Cognition, 33, 1256-1271.

Nosofsky, R. M., \& ZAKI, S. R. (1998). Dissociations between categorization and recognition in amnesic and normal individuals: An exemplar-based interpretation. Psychological Science, 9, 247-255.

Palmeri, T. J., \& Flanery, M. A. (1999). Learning about categories in the absence of training: Profound amnesia and the relationship between perceptual categorization and recognition memory. Psychological Science, 10, 526-530.

Reber, P. J., Gitelman, D. R., Parrish, T. B., \& Mesulam, M. M. (2003). Dissociating explicit and implicit category knowledge with fMRI. Journal of Cognitive Neuroscience, 15, 574-583.

Reed, J. M., Squire, L. R., Patalano, A. L., Smith, E. E., \& Jonides, J. (1999). Learning about categories that are defined by object-like stim- uli despite impaired declarative memory. Behavioral Neuroscience, 113, 411-419.

Shanks, D. R., \& St. John, M. F. (1994). Characteristics of dissociable human learning systems. Behavioral \& Brain Sciences, 17, 367-447.

ZAKI, S. R. (2004). Is categorization performance really intact in amnesia? A meta-analysis. Psychonomic Bulletin \& Review, 11, 10481054.

ZAKI, S. R., \& Nosofsky, R. M. (2001). A single-system interpretation of dissociations between recognition and categorization in a task involving object-like stimuli. Cognitive, Affective, \& Behavioral Neuroscience, 1, 344-359.

(Manuscript received June 27, 2006;

revision accepted for publication October 17, 2006.) 